\title{
Planar chiral meta-materials for optical applications
}

\author{
A. Potts $^{\text {a,b,* }}$, A. Papakostas ${ }^{a}$, D.M. Bagnall ${ }^{\text {a,b }}$, N.I. Zheludev ${ }^{\text {b }}$ \\ ${ }^{a}$ School of Electronics and Computer Science, University of Southampton, Highfield, Southampton SO17 1 BJ, UK \\ ${ }^{\mathrm{b}}$ School of Physics and Astronomy, University of Southampton, Highfield, Southampton SO17 1BJ, UK
}

Available online 19 March 2004

\begin{abstract}
Using electron beam lithography we have fabricated arrays of metallic and non-metallic planar chiral structures on silicon substrates, and observed the pronounced effects of their chirality on the polarization states of the diffracted light in the transmission regime. Significant differences in polarization properties were observed between metallic and polymer structures, most notably in the degree of induced ellipticity for the diffracted light.
\end{abstract}

(c) 2004 Elsevier B.V. All rights reserved.

Keywords: Planar chirality; Optical activity; Nanostructures

\section{Introduction}

Recently a new group of materials has emerged which promise unique polarization characteristics [1]. These planar meta-materials consist of chiral elements that possess no line of symmetry in the plane of the structure. Such structures exhibit twodimensional chirality, the handedness of which is reversed for the mirror image of the structure. Here, we report further results of our research programme to fabricate planar chiral structures, and to study and understand their optical properties.

The ability of left- and right-handed asymmetrical (chiral) three-dimensional helical molecules to rotate the polarization state of light is a fundamental phenomenon in optics as well as having

\footnotetext{
${ }^{*}$ Corresponding author.

E-mail address: ap@esc.soton.ac.uk (A. Potts).
}

important consequences in both chemical and biological systems. However, the concept of chirality also exists in two-dimensions where the chirality is restricted to the asymmetry of objects lying in a plane. So, are these planar media also capable of affecting the polarization states of light? If this were shown to be the case then it could lead to a whole new generation of optical devices and components with potential applications in optoelectronics, telecommunications and nanotechnology. Not surprisingly therefore, there has recently been considerable theoretical interest in this subject, with authors [2-4] proposing planar chiral structures with chirality that could exhibit selective electromagnetic properties. Now, using electron beam lithographic techniques, we have manufactured left and right-handed forms of an artificial medium consisting of microscopic chiral objects distributed regularly over a plane. Optical 
experiments performed previously using a linearly polarized, coherent laser source at a wavelength of $632 \mathrm{~nm}$ showed evidence of handedness-sensitive rotations and elliptization of the polarization state of light diffracted from metallic chiral structures in a reflection configuration [1]. We have now extended these measurements to the study of diffraction in transmitted light at a wavelength of 1520 $\mathrm{nm}$, and compared the results with those derived using non-metallic microstructured chiral films.

\section{Fabrication process}

We have fabricated planar chiral media using two different material systems. Each structure consists of a regular two-dimensional array of microscopic chiral objects in an otherwise homogeneous matrix, each element being in the shape of a gammadion (see Fig. 1). The design length of each section of the gammadion leg was $1.4 \mu \mathrm{m}$, while the bending angle $(\alpha)$ was $45^{\circ}$. Previous experimental work [1] has suggested that gammadions with this bending angle produce the greatest polarization-sensitive results at the wavelengths of interest (632 and $1520 \mathrm{~nm})$. Arrays of both clockwise and anticlockwise gammadions, but of otherwise identical design specifications, were fabricated to provide the two enantiomeric forms of each structure, while the 4-fold rotational symmetry of both the arrays and the gammadions was chosen in order to minimize the optical bire-

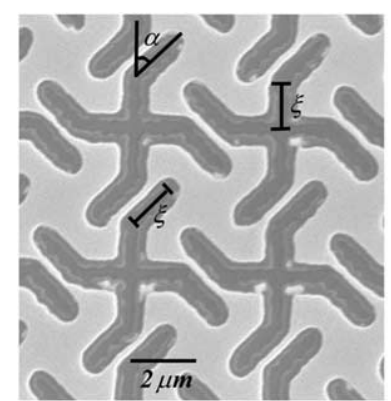

(a)

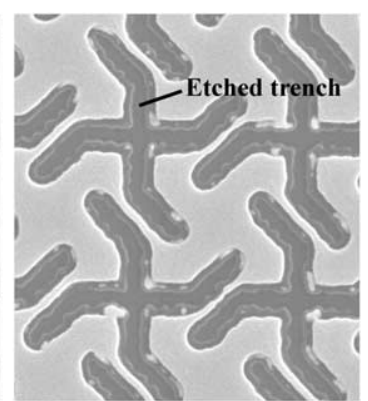

(b)
Fig. 1. SEM image of an array of (a) right-handed and (b) lefthanded chiral gammadion structures etched in a Ti/Au/Ti layer deposited on a double polished silicon substrate. The bending angle of the gammadion arms, $\alpha=45^{\circ}$, while their characteristic length, $\xi=1.4 \mu \mathrm{m}$. fringence of these structures for light at normal incidence.

The samples themselves were manufactured using direct-write electron beam lithography. The fabrication process for the metallic samples has been described previously [5]. First three layers of metal (20 nm Ti, $100 \mathrm{~nm} \mathrm{Au,} 20 \mathrm{~nm} \mathrm{Ti})$ were deposited by electron beam evaporation onto one side of a double-polished silicon wafer. The first layer of titanium was used to improve the adhesion of the much thicker gold layer to the silicon substrate, while the top layer of titanium was used in order to give the layer structure a symmetric profile. The use of double-polished wafers also provides us with the flexibility to perform optical transmission experiments on these structures in the infra-red and microwave regimes.

After deposition, the metallic tri-layer was patterned with arrays of gammadions by electron beam lithography using a $300 \mathrm{~nm}$ layer of high sensitivity UVIII resist. After exposure, the resist was developed for between $60 \mathrm{~s}$ and $2 \mathrm{~min}$ in $\mathrm{Mi}$ croposit 322 developer. The pattern in the developed resist was then transferred to the underlying metal tri-layer using an ion milling process. Finally, the patterned resist mask was removed by plasma etching in oxygen. The final structure (see Fig. 1) consisted of gammadion shaped trenches etched into a Ti/Au/Ti film. The depth of the trenches was sufficient to reveal the underlying silicon substrate.

It should be noted that the ion milling process necessarily results in some widening of the legs of the gammadions due to the gold being etched at a significantly higher rate compared to titanium. In addition, there is also some etching of the underlying silicon substrate. This leads to some irregularity in the etch profile, but this is not believed to have any significant impact on the overall form or magnitude of our observed results.

We have also fabricated chiral structures in thin films of UVIII resist. This was achieved by spinning $300 \mathrm{~nm}$ of resist onto a bare double-polished silicon substrate, and then exposing and developing the resist as described above. The resist structures created in this way were of identical design to the metallic structures. However, because there is no ion milling process step in this case, the man- 
ufactured line-width is much smaller, and closer to the optimum resolution line-width of $200 \mathrm{~nm}$ for our electron beam lithography system, rather than $\sim 600 \mathrm{~nm}$ for the metallic samples.

\section{Optical measurements}

The optical characterization of the metallic chiral gratings was performed using a $\mathrm{HeNe}$ laser at a wavelength of $1520 \mathrm{~nm}$, and the polarization parameters of the transmitted diffracted wave were measured using the 'rotating wave plate' polarimetric technique. The structures were oriented perpendicular to the incident beam, which was linearly polarized, with the polarization direction defined relative to the horizontal axis of the gammadion array. We found that the light diffracted from the structure in the transmission and reflection regimes showed diffraction patterns with well-defined peaks. The polarization states of the diffracted waves were noticeably different from those that would be expected for diffraction from a non-chiral grating. In all of the experiments we analysed the two first-order diffracted beams lying in the plane parallel to the horizontal axis of the gammadion array that also contains the zero-order beam, and measured the difference $\Delta \Theta$, between the polarization azimuth of the incident wave and that of the diffracted beams in transmission. We also measured the ellipticity angle $\eta$ of the diffracted wave $(\eta=0$ corresponds to a linearly polarized wave, with $\eta= \pm 45^{\circ}$ corresponding to right and left circular polarizations accordingly). The polarization azimuth of the incident beam was defined in a clock-wise sense relative to the horizontal axis of the gammadion array.

In all of the arrays described here, the gammadions were designed to have characteristic lengths $\xi=1.4 \mu \mathrm{m}$ and line widths of $w=200 \mathrm{~nm}$ (see Fig. 1). The pitch of the gratings was $4.0 \mu \mathrm{m}$ in both directions while the area of the arrays was designed to be $\approx 1.0 \times 1.0 \mathrm{~mm}^{2}$ so that it was much larger than the diameter of the focused laser beam used for the polarimetric measurements. It should be noted, however, that the manufacturing process generally results in $\xi$ and $w$ being larger than intended, particularly in the metallic samples. This is due to a combination of proximity effects and back-scattering during the electron beam lithography stage, and line width broadening during the ion milling process.

The polarized incident laser beam was orientated normal to the patterned face of the sample and the polarization state of the transmitted beam measured for the two first-order diffracted beams lying in the horizontal plane defined by the horizontal axis of the gammadion array and the zeroorder diffracted beam. The results presented in Fig. 2(a) show that different values of polarization rotation are obtained for the clockwise gammadion array and the anti-clockwise gammadion array, while polarization changes of the diffracted beam

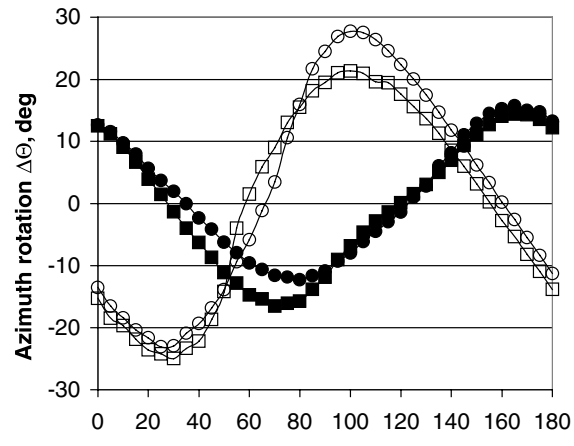

(a)

Azimuth of input polarization $\phi$, deg

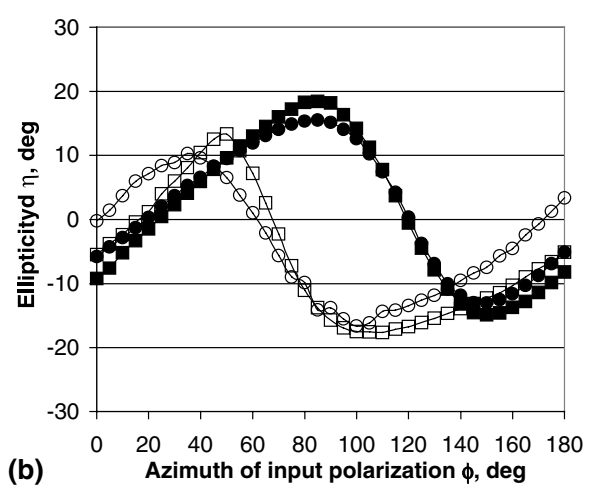

Fig. 2. The polarization azimuth rotation (a) and ellipticity (b) of infra-red light $(1520 \mathrm{~nm})$ diffracted in transmission from arrays of

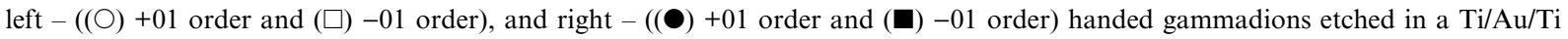
layer, plotted as a function of the azimuth direction of the linearly polarized incident wave. 
observed in mirror-symmetric diffraction orders for the same sample appear almost identical. Both metallic samples induced strong ellipticity in the diffracted beams (see Fig. 2(b)). We attribute the difference in the polarization effect between the two enantiomeric gratings to the chirality of the samples. As we have reported previously [1] such enantiomeric differences may be related to both the "structural" chirality of the sample (i.e., the chirality of arrangement of the individual elements of the structure) and the "molecular" chirality of individual elements. The relative contributions of these two mechanisms will have to be investigated further in the future by comparing with the results of numerical models of diffraction [6]. It is also interesting to note that our attempts to measure the polarization changes in the zero-order transmitted beam failed to show any significant polarization change, thus allowing us to place an upper limit of $\approx 2^{\circ}$ on such effects.

The metallic structures by their nature are inherently lossy due to the substantial imaginary part of the dielectric coefficient in the metal. Dissipation of electromagnetic energy in such structures may be a significant negative factor in their performance. Dielectric chiral structures should be largely free of such losses and could be much more attractive for device applications. We have compared the polarization properties of dielectric structures with their identical metallic counterparts described above. The only significant differ- ences in the topography of the two structures were the thickness of the film $(\sim 300 \mathrm{~nm}$ of UVIII polymer, in comparison with $120 \mathrm{~nm}$ for the metallic film) and the width of the gammadion arms resulting from the fabrication process. In general, the polarization properties of the dielectric gratings were very similar to the metallic gratings. The polarization state of the first order diffracted beams in the transmission regime showed strong enantiomer-specific azimuth rotation (see Fig. 3(a)) and ellipticity (Fig. 3(b)), although the ellipticity was much smaller than in the case of the metallic gratings. This reduction in ellipticity is a useful property of the dielectric gratings and could be a consequence of several factors including the use of loss-less material, the film thickness and the edge profile of the chiral grooves.

\section{Conclusions}

We have fabricated regular 2D arrays of planar chiral elements in metallic and dielectric films that show well defined regular diffraction patterns. These arrays exhibit the capability to alter the polarization state of diffracted light in the transmission regime, with the metallic arrays exhibiting stronger elliptization of the diffracted beams. These changes in the polarization state correlate with each sample's sense of chirality.

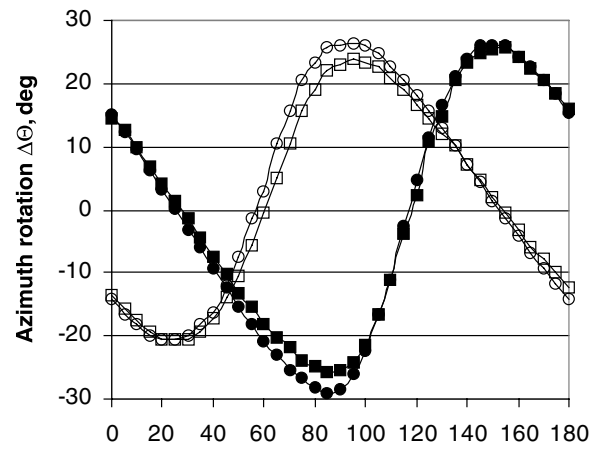

(a) Azimuth of input polarization $\phi$, deg

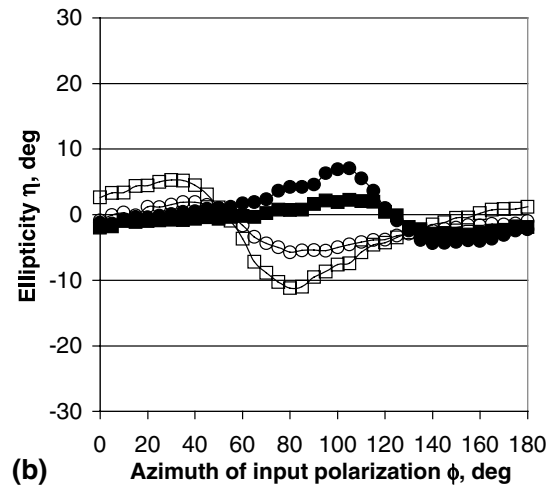

(b)

Fig. 3. The polarization azimuth rotation (a) and ellipticity (b) of infra-red light $(1520 \mathrm{~nm})$ diffracted in transmission from arrays of left $-((\bigcirc)+01$ order and $(\square)-01$ order $)$, and right $-((\mathbf{O})+01$ order and $(\mathbf{\square})-01$ order) handed gammadions etched in a layer of UVIII polymer, plotted as a function of the azimuth direction of the linearly polarized incident wave. 


\section{Acknowledgements}

The authors gratefully acknowledge the EPSRC (UK) for financial support, and S.L. Prosvirnin for useful discussions.

\section{References}

[1] A. Papakostas, A. Potts, D.M. Bagnall, S.L. Prosvirnin, H.J. Coles, N.I. Zheludev, Phys. Rev. Lett. 90 (2003) 107404.
[2] L.R. Arnaut, J. Electromagnet. Wave 11 (1997) 1459.

[3] L.R. Arnaut, L.E. Davis, in: Proceedings of the International Conference on Electromagnetics in Advance Applications, 1995, pp. 381-384.

[4] Y. Svirko, N. Zheludev, M. Osipov, Appl. Phys. Lett. 78 (2001) 498.

[5] A. Potts, A. Papakostas, N.I. Zheludev, H.J. Coles, R. Greef, D.M. Bagnall, J. Mat. Sci.: Mater. Electron. 14 (2003) 393.

[6] S.L. Prosvirnin, N.I. Zheludev, Available from <http:// arxiv.org/abs/ArXive:cond-mat/0309588>, 2003. 